\title{
Extracting contextual information from scientific literature using CERMINE system
}

Dominika Tkaczyk and Łukasz Bolikowski

Interdisciplinary Centre for Mathematical and Computational Modelling, University of Warsaw

\section{Overview}

CERMINE is a comprehensive system for extracting structured metadata and references from born-digital scientific literature. Among other information, the system extracts information related to the context the article was written in, such as the authors and their affiliations, the relations between them or references to other articles. Extracted information is presented in a structured, machine-readable form. CERMINE is based on a modular workflow, whose loosely coupled architecture allows for individual steps evaluation, adjustment or replacement and facilitates future architecture expanding. The implementation is based mostly on supervised and unsupervised machine-learning techniques, which simplifies adapting the system to new document layouts.

\section{Usage}

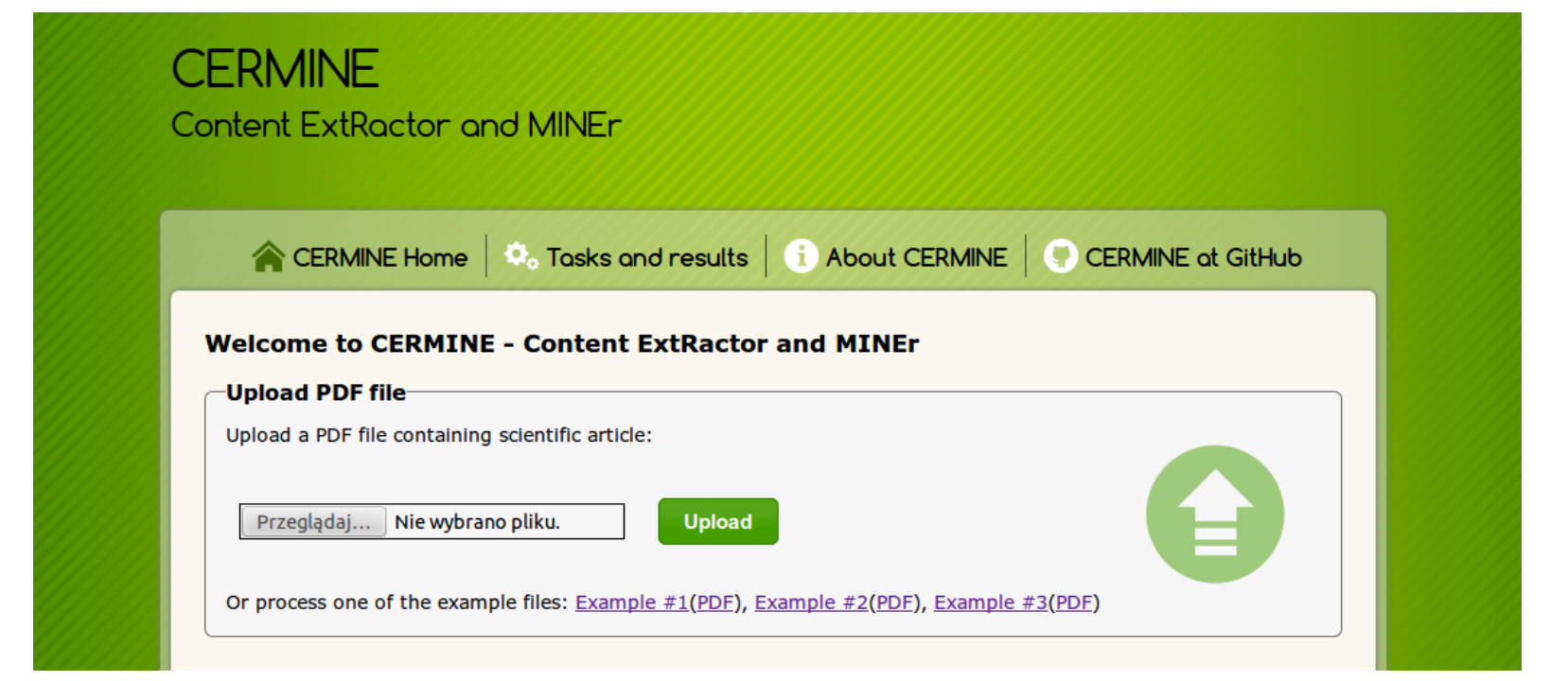

Web service:

cermine.ceon.pl

Open-source code:

github.com/CeON/CERMINE

A RESTful service for executing the extraction process by machines:

\$ curl -X POST --header "Content-Type: application/binary" --data-binary Qarticle.pdf http://cermine.ceon.pl/extract.do

\section{Workflow Architecture}

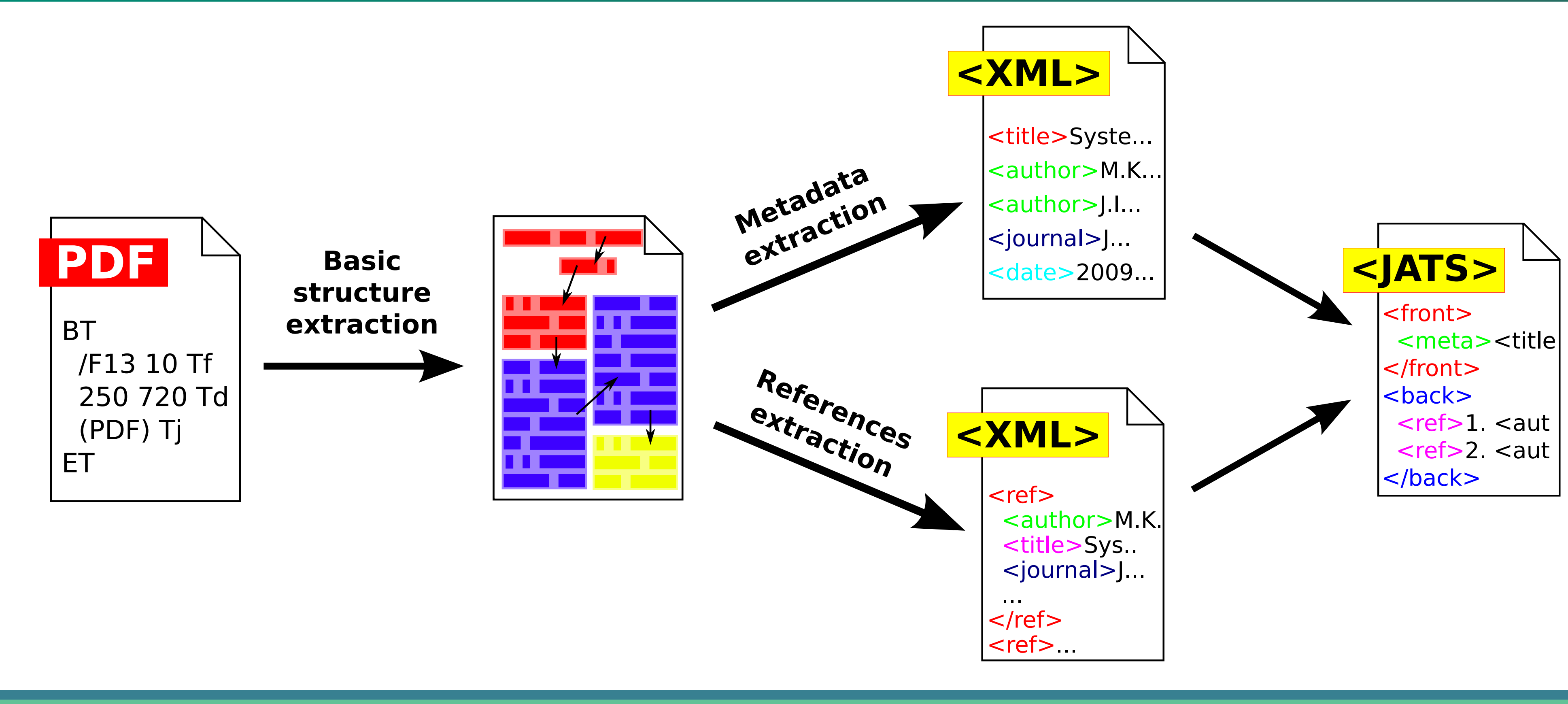

- Basic structure extraction path takes a PDF file on the input and outputs its geometric hierarchical representation, composed of pages, zones, lines, words and characters, along with their coordinates and dimensions, the reading order and general categories (metadata, references, body or other) assigned to zones.

- Metadata extraction path analyses metadata parts of the structure and extracts a rich set of document's metadata, including authors, affiliations and relations between them.

- Bibliography extraction path analyses parts of the structure labelled as references in order to extract a list of parsed citations.

1. Layout Extraction

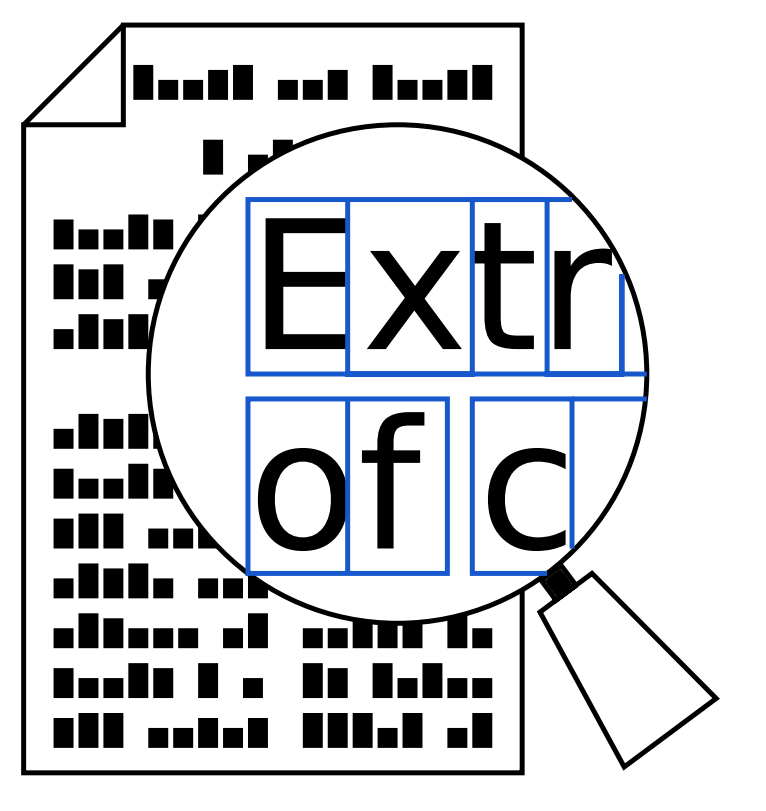

Page

Segmentation:

enhanced

Docstrum

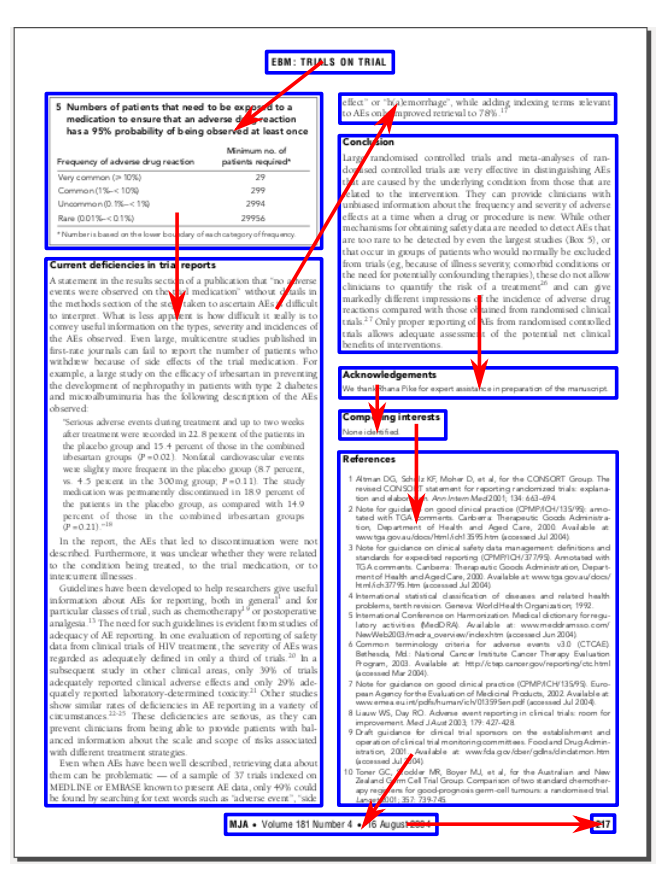

\begin{abstract}
Character
Extraction:

iText library
\end{abstract}

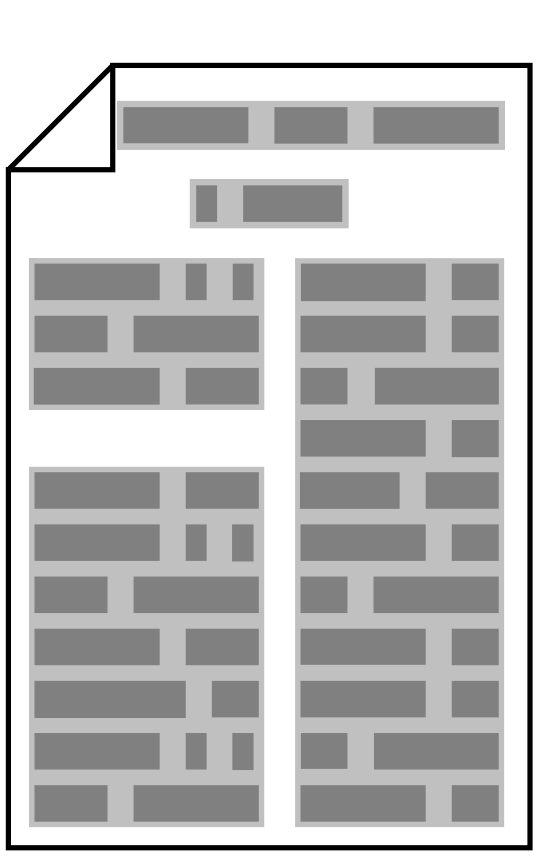

Reading Order

Resolving:

bottom-up

heuristic-based

algorithm

\section{ESWC Challenge}

CERMINE is participating in Task 2 of Semantic Publishing Challenge of ESWC 2015. The system extracts data sufficient for answering queries related to affiliations and citations.

Solving queries Q2.1 (Affiliations in a paper) and Q2.2 (Papers from a country) relies on the features: title, authors and affiliations extraction, establishing author - affiliations relations, detecting country in affiliations.

Solving queries Q2.3 (Cited Works), Q2.4 (Recent Cited Works) and Q2.5 (Cited Journal Papers) relies on features: extracting citations, detecting DOI, title and year in the citation string, recognizing the type of a citation.

The LOD dataset generated by the workflow uses Dublin Core and vCard ontologies and contains resources: volumes, documents, authors, affiliations, countries and citations.

\section{Content Classification}

Content classification is done in two stages:

- initial classification assigns general categories to zones

- metadata classification labels metadata zones with specific metadata classes

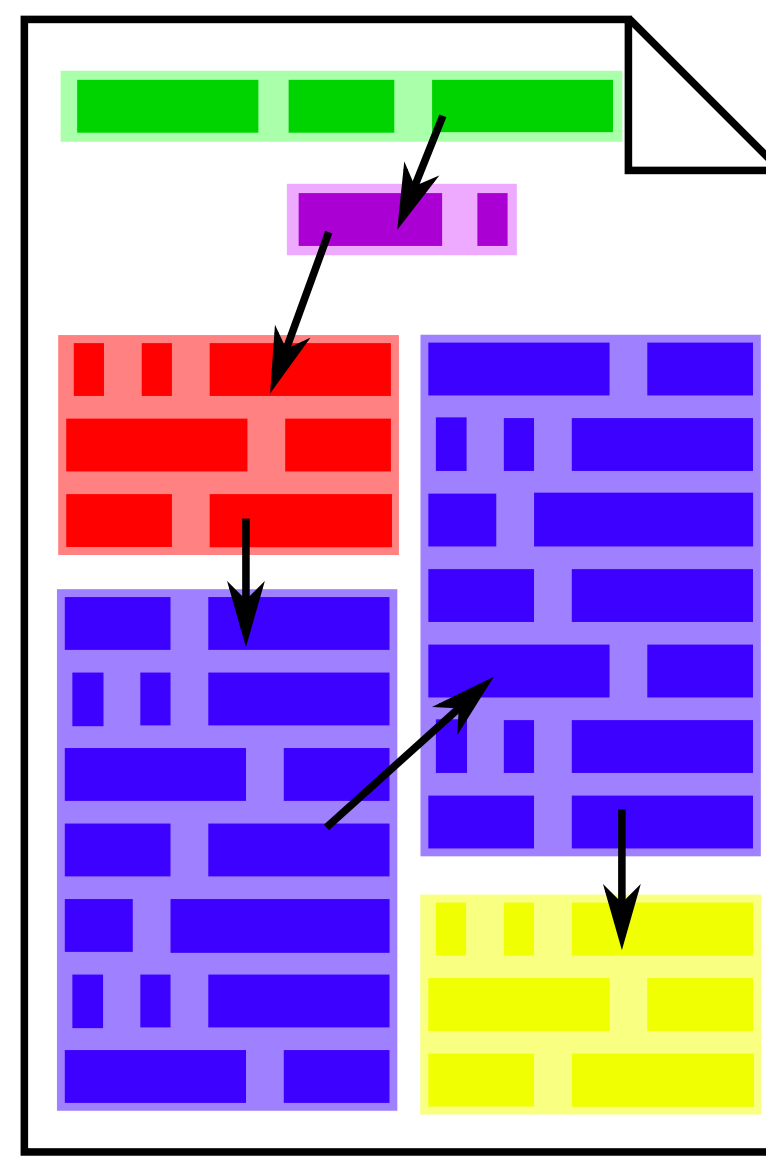

Zones are classified with the use of Support Vector Machines and a rich set of features: geometrical, lexical, sequential, formatting and heuristics-based.

\section{3b. Bibliography Extraction}

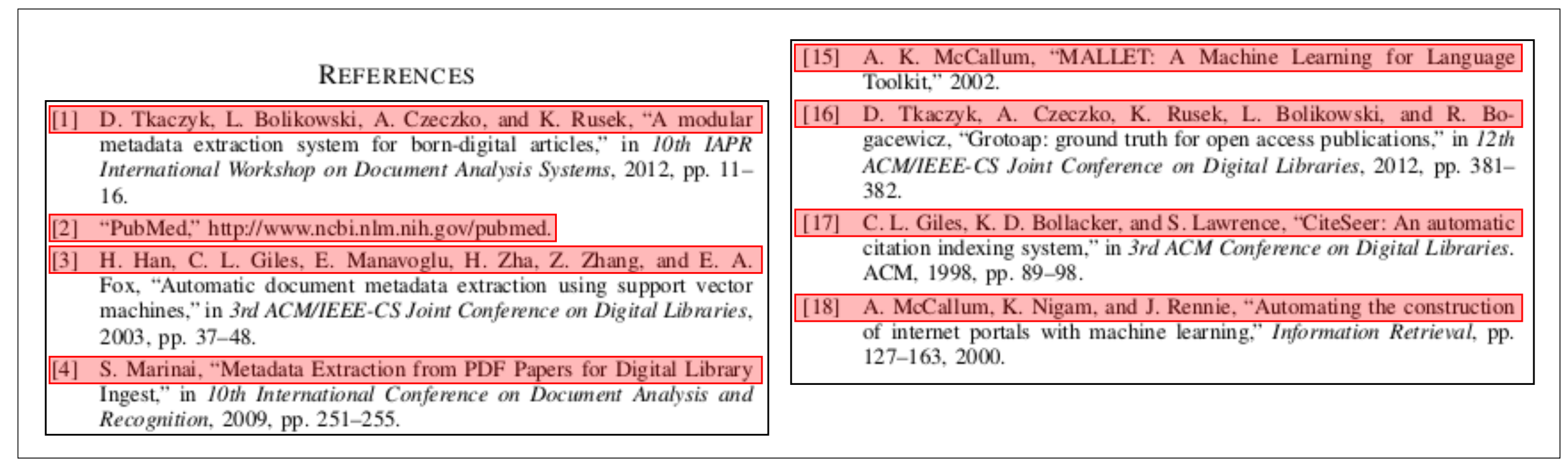

Reference Extraction: extracting individual reference strings using $\mathbf{K}$-Means clustering and both geometric and textual features

[9] L. O'Gorman. The document spectrum for page and Machine Intelligence, 15(11):1162-1173, 1993.

Reference Parsing: extracting metadata from references strings using Conditional Random Fields token classifier and textual features

\section{3a. Affiliation Extraction}

Individual author names, affiliations strings, as well as relations between them are determined using zone labels and heuristics.

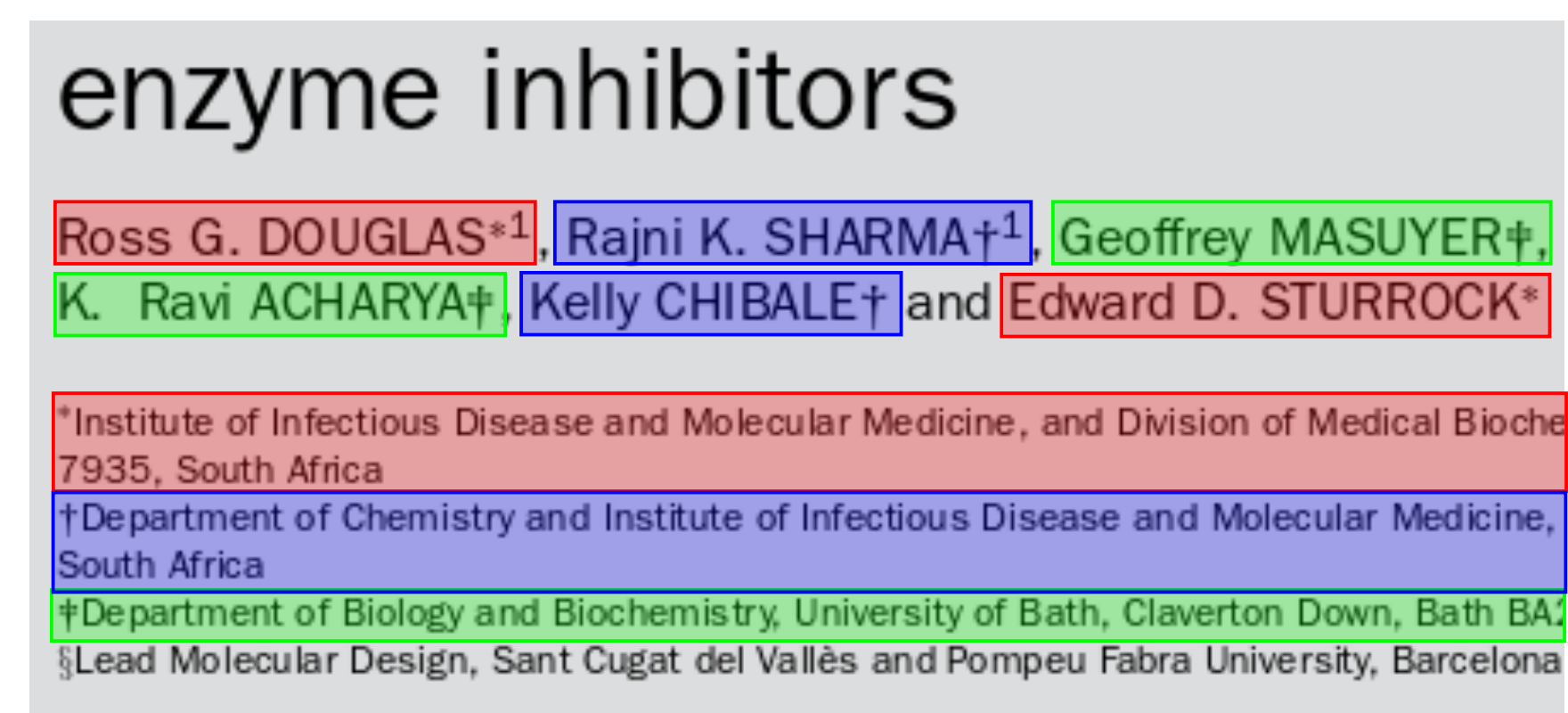

If authors are grouped together in a form of a list, and affiliations are also placed together, the authors are split using a set of separators. Then affiliation indexes are detected based on predefined set of symbols and geometric features. Detected indexes are then used to split affiliation lists and assign affiliations to authors.

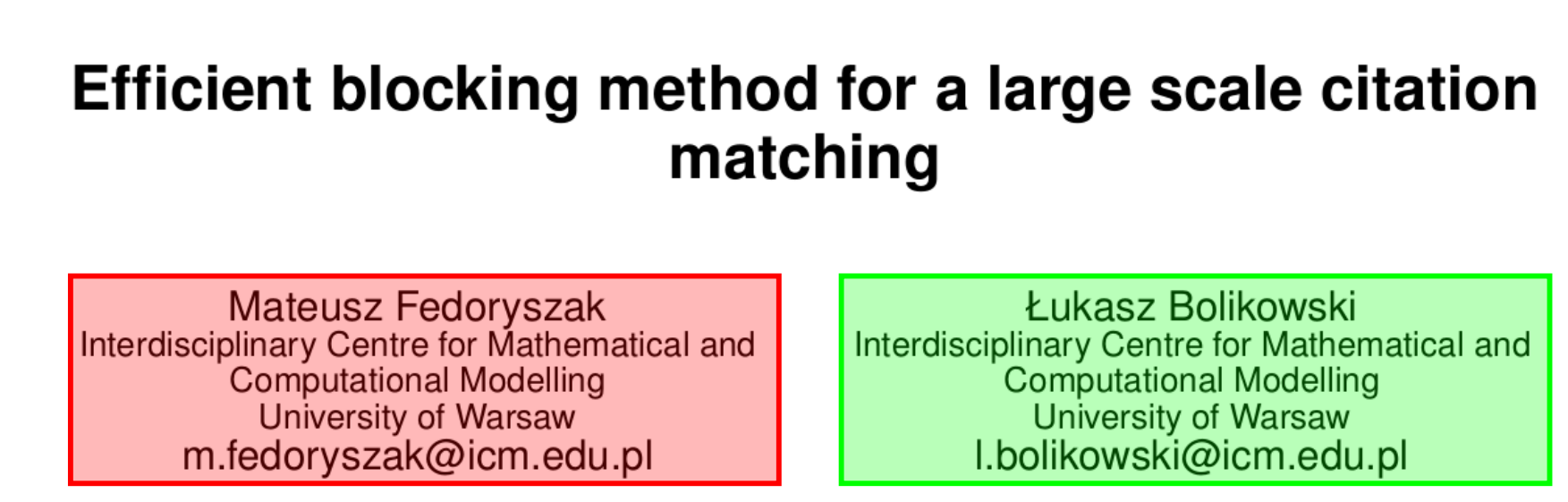

If each author is already assigned to their affiliation by being placed in the same zone, the first line of the zone is treated as the author name, email is detected by a regular expression, and the rest is assumed to be the affiliation string.

Interdisciplinary Centre for Mathematical and Computational Modelling, University of Warsaw,

Affiliation parser uses Conditional Random Fields to detect institution, address and country in affiliation strings.

\section{Find Out More}

